\title{
Blood Chemistry and Major Body Organ Induced-Toxicity by Locally-Made Traditional OMGKRP Karuho Poison in Wistar Albino Rats
}

\author{
Samuel Kule Kyolo', John Odda², Aloysius Lubega², Godfrey S. Bbosa ${ }^{2 *}$ \\ ${ }^{1}$ Department of Pharmacy, Provincial Hospital of North Kivu, Goma, DR. Congo \\ ${ }^{2}$ Department of Pharmacology and Therapeutics, Makerere University College of Health Sciences, Kampala, Uganda \\ Email: ^godfossa@gmail.com
}

How to cite this paper: Kyolo, S.K., Odda, J., Lubega, A. and Bbosa, G.S. (2019) Blood Chemistry and Major Body Organ Induced-Toxicity by Locally-Made Traditional OMGKRP Karuho Poison in Wistar Albino Rats. Neuroscience \& Medicine, 10, 272-291.

https://doi.org/10.4236/nm.2019.103021

Received: August 10, 2019

Accepted: September 21, 2019

Published: September 24, 2019

Copyright $\odot 2019$ by author(s) and Scientific Research Publishing Inc. This work is licensed under the Creative Commons Attribution International License (CC BY 4.0).

http://creativecommons.org/licenses/by/4.0/

\begin{abstract}
OMGKRP is one of various Karuho poison mysteriously used by unscrupulous individuals to kill people during conflict and animals in Goma City, in DRC. The symptoms and signs of most cases are usually confused with many chronic diseases like tuberculosis and HIV/AIDS; with renal, hepatic and cardiac manifestations as well as blood chemistry changes. The study investigated the toxic effect of OMGKRP poison on blood chemistry, serum enzymes and organ toxicity including the kidney, lung, liver and heart of Wistar albino rats. A laboratory-based experimental study was conducted. Fifty animals in 5 groups each with 10 animals were dosed daily for 28 days with 1.0 $\mathrm{mg}, 5.0 \mathrm{mg}, 20.0 \mathrm{mg}$ and $5000.0 \mathrm{mg} / \mathrm{Kg}$ body weight of OMGKRP and normal saline as control group. International standard guidelines, OECD 407 and NIH 2011 were followed during the study period. The blood chemistry analysis, relative organ weight and histopathological changes in the kidney, lung, liver and heart were performed. The findings showed that OMGKRP was associated with increased blood chemistry parameters including total proteins, creatinine, urea, $\mathrm{K}^{+}$levels, direct albumin levels, a decrease in $\mathrm{Cl}^{-}$levels and albumin levels. Histopathological findings showed an increased relative weight and tissue damages of the lung, kidney, liver and heart. Therefore, OMGKRP Karuho poison caused toxicity on blood chemistry, serum enzymes as well as histopathological changes in the lung, renal, hepatic and cardiac tissue damages in Wistar albino rats.
\end{abstract}

\section{Keywords}

Blood Chemistry, Serum Enzymes, OMGKRP Karuho Poison, Organ Toxicity, Sub-Acute Toxicity 


\section{Introduction}

Poisoning of human and animals is a global health problem. The World Health Organization (WHO) estimates that about 0.3 million people die every year due to various poisonous agents in the world [1]. In most countries, accidental poisoning with drugs used in pharmacotherapy has been reported as commonest form of poisoning. However, in DRC, intentional poisonings seem to be a common form of murder using Karuho poison that is usually a mixture of two or more substances especially made from some reptiles like the Chameleon or lizards, toads/frogs, human placenta, Amanita species of mushroom, Datura species of plants heavy metals including arsenic cadmium and mercury [2], glass powders and many others are used. The practice of Karuho poisoning is very common in the different regions of DRC that are affected by war and conflicts such as Goma city and its surrounding areas that have been affected by various types of homicide ranging from army confrontations, rape, massacre, poisoning and war crimes [3]. According to popular belief in North Kivu province, Karuho poisoning is practiced by unscrupulous individuals who incorporate it in the food or beverages which is intended for a victim. The signs and symptoms are usually similar to chronic diseases like tuberculosis (TB), HIV/AIDS [2] and many organ pathologies. In the local communities of Goma city, the phobia of Karuho poison is a real social threat and has changed the behavior of people towards their neighbors and treatment seeking patterns for many conditions including TB and HIV/AIDS [2]. The suspicion of each other, are noticed as consequences of social behavioral change in communities. Since Karuho poisoning has similar clinical symptoms to TB, HIV/AIDS, typhoid fever, malaria and various types of cancer, so many people first consult traditional healers when they contract these diseases because they have tendency to believe that they were poisoned by Karuho. It is reported that more than 30 percent of TB diagnosed cases in health care centres in Eastern DRC had initially thought to have been poisoned by Karuho poison according to Bahabo [4]. Usually the poison is mixed in food and drinks in small amounts which is then consumed by the victim thus causing a slow progressive damage to the tissues and organs of the affected individual (sub-acute toxicity).

Various compounds in Karuho poison that is made locally from different natural products [5] exists including human placenta, Zetekitoxin $\mathrm{AB}$ compound from Panamanian golden toad [6] [7], bufadienolides [6] [7] such as bufalotoxin, bufagins and bufotenin compounds from the parotid gland and skin of the Bufalo genus of toads [8], Digitalis [9] [10], hemorrhagins like gilatoxin from the venoms of Helodermatidae lizard family [11]-[16]. However, the Helodermatid lizard venoms have been reported to be antigenically distinct from snake venoms and exhibiting marked thermostability, retaining toxicity after autoclaving at $100^{\circ} \mathrm{C}$ for 20 minutes [17]. Also tropane alkaloids like scopolamine, hyoscyamine, and atropine from Datura stramonium have been reported to be used [18] [19]. In addition, alpha-amanitin and phalloidin compounds from the genus Amanita mushroom [20] [21] are used. Heavy metals including, arsenic, cad- 
mium and mercury have a high degree of toxicity have also been reported to be used in the preparation of the Karuho poison [22]. In addition, glass powder is also used in the preparation of the poison. Also several arsenic-containing manufactured products such as insecticides, herbicides, sheep dips, and dyestuff are available in communities within Goma City which can be a source of arsenicals to the unscrupulous herbalists in their preparation of Karuho poison. Once the victim is exposed to this kind of poison, they exhibit the signs and symptoms that are similar to those of TB and HIV/AIDS. However, there is limited information on the effects of Karuho poison on blood chemistry, serum enzymes and organ toxicity. The study determined the toxic effects of OMGKRP Karuho on blood chemistry, serum enzymes as well as histopathological changes in the lung, renal, hepatic and cardiac tissue in Wistar albino rats.

\section{Materials and Methods}

\subsection{Study Design}

It was an experimental-based study that determined the toxic effects of OMGKRP Karuho on blood chemistry, serum enzymes as well as histopathological changes in the lung, renal, hepatic and cardiac tissue damages in Wistar albino rats.

\subsection{Study Setting}

The OMGKRP samples were purchased from traditional herbalist in Goma City in DRC and the laboratory experiments were carried out at the department of Pharmacology \& Therapeutics, Makerere University College of Health Sciences (MakCHS) and at the department of Veterinary Pathology, Makerere University College of Veterinary Medicine, Animal Resources and Bio-security (MakCOVAB), Kampala, Uganda.

\subsection{Selection Criteria of OMGKRP Karuho Poison}

The OMGKRP Karuho poison was selected based on its potency or activity using the previous study findings during the screening study that was conducted to determine the toxicity profile of the different Karuho poisons and their effects on the brain of Wistar rats [23].

\subsection{Purchasing, and Handling of OMGKRP Karuho Poison Samples}

Four samples of OMGKRP were purchased from a well-known traditional healer who provided the same sample for previous study [23] and who prepared it using the same ingredients. However, the healer kept secret, the ingredients used in the preparation of the OMGKRP poison. Once purchased, samples were sealed in the dry, opaque and closed plastic container before taken to the department of Pharmacology and Therapeutics, where the study was conducted. They were kept at temperatures between $2^{\circ} \mathrm{C}-8^{\circ} \mathrm{C}$, under lock and away from 
research staff. Any handling was done according to good laboratory practice (GLP) [24].

\subsection{Selection and Handling of Experimental Laboratory Animals}

A total of 50 adult Wistar albino rat males and females aged between $8-12$ weeks and weighting $180 \pm 20 \mathrm{~g}$, bred in COVAB animal house were randomly selected using the computer (statistical package MINITAB). The purchased and selected rats were housed in animal house at the department of Pharmacology and Therapeutics, MakCHS for 1 week for acclimatization. They were housed under standard environmental conditions, at temperature of $20^{\circ} \mathrm{C}-24^{\circ} \mathrm{C}$; light/dark, hour cycle of $14 / 10$; relative humidity of $65 \%$, given free access to food, water and in mini-cage size of $350 \mathrm{~cm}^{2}$ for rats with freedom of movement [25] [26]. They were fed on commercial rat feed pellet that was purchased from Uganda Grain Miller in Kampala city and with water ad-libitum [27].

\subsection{Selection Criteria of the Animals}

Both males and females animals which were normal as judged by their alertness and between 8 and 12 weeks of age were selected for the study. Sick, pregnant animals and nursing mothers were excluded in this study basing on the ethical guidelines on the Laboratory use of animals in bio-medical research [26].

\subsection{Experimental Procedure}

\subsubsection{Preparation of Crude Aqueous Suspension of OMGKRP}

About $10 \mathrm{~g}$ of OMGKRP sample was weighted using analytical balance. Using graduate cylinder or conical flask and glass rod, sample was mixed with distilled water up to 100 milliliters to make a stock suspension of $100 \mathrm{mg} / \mathrm{ml}$ from which suitable dilutions were made in varying doses. Controls received distilled water. The doses were administered as $\mathrm{mg} / \mathrm{Kg}$ body weight (bwt) of each rat.

\subsubsection{Determination of Sub-Acute Effect of OMGKRP on Kidney, Lung, Liver and Heart Tissues}

Three dose levels including 1, 5 and $20 \mathrm{mg} / \mathrm{Kg}$ bwt were selected from acute toxicity study carried out in previous study [23] and were administered daily to the rats for 28-days according to OECD 407 methods [28] using the intra-gastric tube directly into the stomach. Unexceptional dose of $5000 \mathrm{mg} / \mathrm{Kg}$ was used as fourth dose as a way to compare its toxic effects to that of the lowest dose that is commonly used by unscrupulous individuals. Thus, rats were randomly divided into five groups with each having 10 animals of both sexes in equal number for each group. The animals were starved overnight prior to administration of OMGKRP doses. The tested substance and distilled water were orally administered daily by intra-gastric tube in graduated dose levels once daily for a period of 28 days [28]. Food was withheld for 3 - 4 hours after administration of Karuho poison and for the control group. Thereafter animal had free access to food and water. 


\subsubsection{Blood Chemistry Analysis}

About $2 \mathrm{ml}$ of blood samples were collected in the red top vacutainers free from anticoagulant after sacrificing them on Day 28 . The collected blood samples were centrifuged for ten minutes at $10,000 \mathrm{rpm}$ at room temperature $\left(25^{\circ} \mathrm{C}\right)$ to obtain serum samples. The serum chemistry parameters including total protein, albumin, urea, creatinine, potassium, $\mathrm{Na}^{+}$and $\mathrm{Cl}^{-}$levels were analyzed at Clinical Chemistry Laboratory at Mulago National Referral Hospital using Cobas Integra 400 Clinical Chemistry Analyzer using standard methods and procedures.

\subsubsection{Relative Organ Weight (ROW) Determination}

Individual vital organs including kidneys, lungs, liver, and heart were collected immediately, mopped with filter paper and weighed using laboratory analytical balance and the relative weights were calculated using the formula below and expressed as $\mathrm{g} / 100 \mathrm{~g}$ bwt.

$$
\text { ROW }=\frac{\text { Absolute organ weight }(g)}{\text { Body weight of rat on sacrifice day }(g)} \times 100
$$

\subsubsection{Histopathological Analysis of Kidney, Lung, Liver and Heart Tissues of Wistar Albino Rats}

The animal was put to sleep by injecting $30 \mathrm{mg} / \mathrm{Kg}$ bwt of sodium pentobarbitone to minimize pain and harm to the animal. The procedure was conducted at the Department of Pharmacology and Therapeutics and the histopathological analysis was done at the Department of Pathology at COVAB, Makerere University. The kidney, lung, liver and heart of sacrificed animals were transferred to $4 \%$ formalin solution for fixation and later on, processed for histopathological studies following the standard procedure described by Raghuramulu et al. (1983) [29]. The microtome sections were made, processed and stained with haematoxylin and eosin. The sections of kidney, lung, liver and heart were mounted on the slide and viewed using binocular research Nikon Eclipse Ci-S microscope (Japan) under different magnifications. Changes, if any in the organ cyto-architecture were noted. Also, toxic effects of OMGKRP were deducted from blood chemistry parameters and relative organ weight of Wistar rats [30].

\subsection{Data Analysis}

Quantitative data were analyzed using SPSS Version 20 statistical package. Two-way ANOVA was used to compare means within and between groups. A p-value of $<0.05$ was considered statistically significant. Also, changes in organs cyto-architecture were revealed by photomicrography analysis using comparison between control kidney, lung, liver and heart tissue (normal tissue) and treated rats kidney, lung, liver and heart tissues.

\subsection{Ethical Consideration}

Ethical approval to conduct the study was sought from the Department of Pharmacology and Therapeutics, MakCHS, School of Biomedical Sciences Higher 
degrees Research and Ethics Committee (IRB) with approval number SBS 346, and Research and Ethics Committee of ULPGL. The study was carried out according to the international standard guidelines on use of laboratory animals in biomedical research [25] [27].

\section{Results}

The findings from the study show that none of the animals died after administration of the different dose levels of the OMGKRP Karuho poison within 24 hours of administration (no acute toxicity observed) and that is why also in the communities the victims do not die immediately. However, the following signs and symptoms were observed overtime following the administration of the different dose levels of the poison and these included body weight loss, shortness of breath, reduced food and water intake and piloerection. These signs and symptoms were similar to what is observed among the victims in the communities when exposed to these Karuho poisons and therefore they are commonly confused with signs of tuberculosis, typhoid, HIV/AIDS and many other conditions.

\subsection{Blood Chemistry Analysis}

The treated male and female rats with different doses of OMGKRP crude aqueous suspension showed significant differences from control groups in total protein levels $(1.0 \mathrm{mg} / \mathrm{Kg}, \mathrm{p}=0.004 ; 5.0 \mathrm{mg} / \mathrm{Kg}, \mathrm{p}=0.02$ and $20.0 \mathrm{mg} / \mathrm{Kg}, \mathrm{p}<$ $0.001)$, albumin $(5000.0 \mathrm{mg} / \mathrm{Kg}, \mathrm{p}=0.02)$, urea (ANOVA values $=0.04)$, creatinine (ANOVA value $=0.03)$, potassium $(20.0 \mathrm{mg} / \mathrm{Kg}, \mathrm{p}=0.009)$ and chloride levels $(5000.0 \mathrm{mg} / \mathrm{Kg}, \mathrm{p}=0.006)$. The treated male rats showed significant difference from control group in total proteins $(1.0 \mathrm{mg} / \mathrm{Kg}, \mathrm{p}=0.03 ; 5.0 \mathrm{mg} / \mathrm{Kg}, \mathrm{p}$ $=0.004 ; 20.0 \mathrm{mg} / \mathrm{Kg}, \mathrm{p}=0.001)$, direct bilirubin $(20.0 \mathrm{mg} / \mathrm{Kg}, \mathrm{p}=0.04)$ and chloride levels $(5000.0 \mathrm{mg} / \mathrm{Kg}, \mathrm{p}=0.02)$ (Table 1$)$. The treated female rats showed significant difference from control group in total proteins levels (1.0 $\mathrm{mg} / \mathrm{Kg}, \mathrm{p}=0.03$ and $20.0 \mathrm{mg} / \mathrm{Kg}, \mathrm{p}=0.01)$ and albumin levels $(5000.0 \mathrm{mg} / \mathrm{Kg}, \mathrm{p}$ $=0.03)$ (Table 1). However, there was no statistically significant difference as compared to the control group in sodium levels. There was dose-depended change (increase) on GGT, ALT and AST levels in treated group without statistically significant difference as compared to the control group. ALP levels presented no statistically significant difference in all treated group as compared to control group. The maximum effect (increase) was observed at the dose of 20.0 $\mathrm{mg} / \mathrm{Kg}$ bwt for almost all parameters including serum creatinine, total proteins, urea, and potassium levels. However, the effect of OMGKRP on serum albumin, AST, ALT and chloride levels was observed at highest dose level of $5000.0 \mathrm{mg} / \mathrm{Kg}$ bwt (Table 1). However, it was observed that OMGKRP Karuho poison was able to cause serious effects on the clinical chemistry parameters in low dose levels especially at $20.0 \mathrm{mg} / \mathrm{Kg}$ bwt and not the $5000.0 \mathrm{mg} / \mathrm{Kg}$ bwt limit dose and this is true since some poisons always cause severe effects in more dilute form than the concentrated form (Table 1). 
Table 1. Effect of repeated oral dose of OMGKRP on clinical chemistry parameters of Wistar albino rats after the 28th day of treatment.

\begin{tabular}{|c|c|c|c|c|}
\hline \multicolumn{2}{|c|}{ Blood chemistry parameters } & \multicolumn{3}{|c|}{ Values } \\
\hline & Group & Both male \& female & Male & Female \\
\hline \multicolumn{5}{|l|}{ Albumin (g/dL) } \\
\hline & Control & $39.69 \pm 1.36$ & $37.70 \pm 0.50$ & $42.27 \pm 2.57$ \\
\hline & $1 \mathrm{mg} / \mathrm{Kg}$ bwt & $40.31 \pm 0.92(0.99)$ & $39.23 \pm 1.32(0.89)$ & $41.77 \pm 0.74(1.00)$ \\
\hline & $5 \mathrm{mg} / \mathrm{Kg}$ bwt & $40.11 \pm 0.96(1.00)$ & $39.52 \pm 0.87(0.82)$ & $40.90 \pm 2.10(0.98)$ \\
\hline & $20 \mathrm{mg} / \mathrm{Kg}$ bwt & $41.10 \pm 0.82(0.86)$ & $40.90 \pm 1.22(0.31)$ & $41.43 \pm 1.09(1.00)$ \\
\hline & 5000 mg/Kg bwt & $33.95 \pm 1.46\left(\mathbf{0 . 0 2} \mathbf{2}^{\star}\right)$ & $35.65 \pm 3.61(0.83)$ & $32.25 \pm 0.75\left(0^{0.03^{\star}}\right)$ \\
\hline & Ref. range & $34-55$ & $34-48$ & $36-55$ \\
\hline & ANOVA p-value & $0.004^{*}$ & 0.12 & $0.03^{*}$ \\
\hline \multicolumn{5}{|l|}{ CREA $(\mu \mathrm{mol} / \mathrm{L})$} \\
\hline & Control & $49.43 \pm 2.33$ & $49.25 \pm 2.50$ & $49.67 \pm 5.04$ \\
\hline & $1 \mathrm{mg} / \mathrm{Kg}$ bwt & $62.14 \pm 5.71(0.39)$ & $60.75 \pm 7.11(0.78)$ & $64.00 \pm 11.15(0.76)$ \\
\hline & $5 \mathrm{mg} / \mathrm{Kg}$ bwt & $57.88 \pm 5.19(0.73)$ & $55.00 \pm 6.65(0.98)$ & $60.75 \pm 8.73(0.86)$ \\
\hline & $20 \mathrm{mg} / \mathrm{Kg}$ bwt & $65.75 \pm 5.61(0.15)$ & $66.80 \pm 8.38(0.39)$ & $64.00 \pm 5.57(0.76)$ \\
\hline & 5000 mg/Kg bwt & $40.75 \pm 4.03(0.83)$ & $42.50 \pm 9.50(0.98)$ & $39.00 \pm 1.00(0.93)$ \\
\hline & Ref. range & $20-60$ & $20-50$ & $20-60$ \\
\hline & ANOVA p-value & $0.03^{*}$ & 0.25 & 0.32 \\
\hline \multicolumn{5}{|l|}{$\operatorname{ALP}(\mathrm{U} / \mathrm{L})$} \\
\hline & Control & $202.42 \pm 20.45$ & $191.53 \pm 36.11$ & $213.30 \pm 26.63$ \\
\hline & $1 \mathrm{mg} / \mathrm{Kg}$ bwt & $179.59 \pm 38.08(0.99)$ & $192.33 \pm 49.73(1.00)$ & $162.60 \pm 70.36(0.97)$ \\
\hline & $5 \mathrm{mg} / \mathrm{Kg}$ bwt & $205.68 \pm 46.01(1.00)$ & $203.88 \pm 62.13(1.00)$ & $207.48 \pm 77.56(1.00)$ \\
\hline & $20 \mathrm{mg} / \mathrm{Kg}$ bwt & $176.73 \pm 20.03(0.98)$ & $176.82 \pm 32.97(1.00)$ & $176.57 \pm 11.03(0.99)$ \\
\hline & $500 \mathrm{mg} / \mathrm{Kg}$ bwt & $170.15 \pm 28.67(0.98)$ & $190.00 \pm 63.80(1.00)$ & $149.40 \pm 0.70(0.96)$ \\
\hline & Ref. range & $26-230$ & $62-230$ & $26-147$ \\
\hline & ANOVA p-value & 0.93 & 1.00 & 0.93 \\
\hline \multicolumn{5}{|l|}{ GGT (U/L) } \\
\hline & Control & $2.91 \pm 2.50$ & $2.13 \pm 3.54$ & $3.97 \pm 4.21$ \\
\hline & $1 \mathrm{mg} / \mathrm{Kg}$ bwt & $1.59 \pm 1.23(1.00)$ & $2.83 \pm 1.86(1.00)$ & $0.07 \pm 1.10(0.97)$ \\
\hline & $5 \mathrm{mg} / \mathrm{Kg}$ bwt & $4.40 \pm 2.23(0.99)$ & $2.13 \pm 1.25(1.00)$ & $6.68 \pm 4.27(0.99)$ \\
\hline & $20 \mathrm{mg} / \mathrm{Kg}$ bwt & $8.41 \pm 3.30(0.51)$ & $6.52 \pm 4.02(0.83)$ & $11.57 \pm 6.33(0.73)$ \\
\hline & 5000 mg/Kg bwt & $6.90 \pm 2.10(0.87)$ & $7.55 \pm 3.15(0.85)$ & $6.25 \pm 3.95(1.00)$ \\
\hline & Ref. range & $0.00-1.00$ & $0.00-1.00$ & $0.00-0.40$ \\
\hline & ANOVA p-value & 0.31 & 0.69 & 0.50 \\
\hline \multicolumn{5}{|l|}{$\operatorname{ALT}(\mathrm{U} / \mathrm{L})$} \\
\hline & Control & $137.00 \pm 24.64$ & $161.28 \pm 40.43$ & $104.63 \pm 7.96$ \\
\hline
\end{tabular}




\begin{tabular}{|c|c|c|c|c|}
\hline & $1 \mathrm{mg} / \mathrm{Kg}$ bwt & $137.21 \pm 22.11(1.00)$ & $165.85 \pm 29.91(1.00)$ & $99.03 \pm 18.86(1.00)$ \\
\hline & $5 \mathrm{mg} / \mathrm{Kg}$ bwt & $149.19 \pm 33.32(1.00)$ & $121.88 \pm 21.33(1.00)$ & $176.1 \pm 43.4(0.86)$ \\
\hline & $20 \mathrm{mg} / \mathrm{Kg}$ bwt & $152.01 \pm 33.01(1.00)$ & $171.74 \pm 47.17(1.00)$ & $119.1 \pm 43.3(1.00)$ \\
\hline & $500 \mathrm{mg} / \mathrm{Kg}$ bwt & $221.65 \pm 60.60(0.53)$ & $207.90 \pm 87.10(0.96)$ & $235.4 \pm 118.6(0.59)$ \\
\hline & Ref. range & $16-48$ & $18-45$ & $16-48$ \\
\hline & ANOVA p-value & 0.56 & 0.80 & 0.50 \\
\hline \multicolumn{5}{|l|}{ AST (U/L) } \\
\hline & Control & $281.6 \pm 87.5$ & $347.2 \pm 106.2$ & $183.1 \pm 162.8$ \\
\hline & $1 \mathrm{mg} / \mathrm{Kg}$ bwt & $370.2 \pm 77.2(0.99)$ & $421.0 \pm 119.3(0.96)$ & $293.9 \pm 84.6(1.00)$ \\
\hline & $5 \mathrm{mg} / \mathrm{Kg}$ bwt & $415.43 \pm 133.44(0.97)$ & $314.5 \pm 113.1(0.98)$ & $516.4 \pm 271.4(0.90)$ \\
\hline & $20 \mathrm{mg} / \mathrm{Kg}$ bwt & $507.8 \pm 147.4(0.78)$ & $543.5 \pm 264.1(0.75)$ & $454.4 \pm 56.8(0.95)$ \\
\hline & $500 \mathrm{mg} / \mathrm{Kg}$ bwt & $631.8 \pm 261.4(0.47)$ & $475.0 \pm 315.0(0.98)$ & $788.7 \pm 511.4(0.57)$ \\
\hline & Ref. range & $65-203$ & $74-143$ & $65-203$ \\
\hline & ANOVA p-value & 0.52 & 0.92 & 0.61 \\
\hline \multicolumn{5}{|l|}{$\mathrm{TP}(\mathrm{g} / \mathrm{dL})$} \\
\hline & Control & $7.08 \pm 0.08$ & $7.12 \pm 0.042$ & $7.03 \pm 0.20$ \\
\hline & $1 \mathrm{mg} / \mathrm{Kg}$ bwt & $7.93 \pm 0.19\left(0.004^{*}\right)$ & $7.68 \pm 0.12\left(0.03^{*}\right)$ & $8.27 \pm 0.36\left(\mathbf{0 . 0 3}^{*}\right)$ \\
\hline & $5 \mathrm{mg} / \mathrm{Kg}$ bwt & $7.77 \pm 0.13(\mathbf{0 . 0 2 *})$ & $7.88 \pm 0.12\left(0.004^{*}\right)$ & $7.60 \pm 0.24(0.37)$ \\
\hline & $20 \mathrm{mg} / \mathrm{Kg}$ bwt & $8.17 \pm 0.14\left(<0.0001^{* * *}\right)$ & $7.97 \pm 0.14\left(0.001^{*}\right)$ & $8.50 \pm 0.15\left(0.01^{*}\right)$ \\
\hline & 5000 mg/Kg bwt & $6.65 \pm 0.28(0.45)$ & $7.13 \pm 0.08(1.00)$ & $6.18 \pm 0.35(0.27)$ \\
\hline & Ref. range & $5.2-7.7$ & $5.2-7.1$ & $5.5-7.7$ \\
\hline & ANOVA p-value & $<0.0001^{* * *}$ & $<0.0001^{* * *}$ & $0.001^{* * *}$ \\
\hline \multicolumn{5}{|l|}{ Urea (mmol/L) } \\
\hline & Control & $7.76 \pm 0.49$ & $6.91 \pm 0.20$ & $8.88 \pm 0.69$ \\
\hline & $1 \mathrm{mg} / \mathrm{Kg}$ bwt & $10.17 \pm 1.07(0.31)$ & $9.79 \pm 1.42(0.18)$ & $10.67 \pm 1.95(0.94)$ \\
\hline & $5 \mathrm{mg} / \mathrm{Kg} \mathrm{bwt}$ & $9.85 \pm 0.51(0.42)$ & $9.76 \pm 0.93(0.18)$ & $9.94 \pm 0.59(1.00)$ \\
\hline & $20 \mathrm{mg} / \mathrm{Kg}$ bwt & $11.21 \pm 1.19\left(0.05^{\star}\right)$ & $10.06 \pm 0.47(0.09)$ & $13.13 \pm 3.10(0.45)$ \\
\hline & 5000 mg/Kg bwt & $7.77 \pm 0.42(1.00)$ & $7.76 \pm 0.80(0.99)$ & $7.88 \pm 0.64(1.00)$ \\
\hline & Ref. range & $4.43-9.76$ & $4.43-8.86$ & $4.75-9.76$ \\
\hline & ANOVA p-value & $0.04^{*}$ & 0.07 & 0.37 \\
\hline \multicolumn{5}{|l|}{$\operatorname{DBil}(\mu \mathrm{mol} / \mathrm{L})$} \\
\hline & Control & $1.65 \pm 0.21$ & $1.37 \pm 0.19$ & $1.93 \pm 0.33$ \\
\hline & $1 \mathrm{mg} / \mathrm{Kg}$ bwt & $2.07 \pm 0.11(1.00)$ & $1.95 \pm 0.18(0.70)$ & $2.23 \pm 0.09(1.00)$ \\
\hline & $5 \mathrm{mg} / \mathrm{Kg}$ bwt & $2.24 \pm 0.23(1.00)$ & $1.88 \pm 0.27(0.79)$ & $2.60 \pm 0.29(1.00)$ \\
\hline & $20 \mathrm{mg} / \mathrm{Kg}$ bwt & $4.86 \pm 2.33(0.31)$ & $2.85 \pm 0.47\left(0.04^{*}\right)$ & $7.53 \pm 5.59(0.54)$ \\
\hline & $5000 \mathrm{mg} / \mathrm{Kg}$ bwt & $1.48 \pm 0.23(1.00)$ & $1.25 \pm 0.25(1.00)$ & $1.70 \pm 0.40(1.00)$ \\
\hline & Ref. range & $0.51-1.03$ & $0.51-0.85$ & $0.51-1.03$ \\
\hline & ANOVA p-value & 0.25 & $0.003^{*}$ & 0.50 \\
\hline
\end{tabular}




\section{Continued}

$\mathrm{Na}^{+}(\mu \mathrm{mol} / \mathrm{L})$

$$
\begin{gathered}
\text { Control } \\
1 \mathrm{mg} / \mathrm{Kg} \text { bwt } \\
5 \mathrm{mg} / \mathrm{Kg} \text { bwt } \\
20 \mathrm{mg} / \mathrm{Kg} \text { bwt } \\
5000 \mathrm{mg} / \mathrm{Kg} \text { bwt } \\
\text { Ref. range }
\end{gathered}
$$$$
\text { ANOVA p-value }
$$

$\mathrm{K}^{+}(\mu \mathrm{mol} / \mathrm{L})$

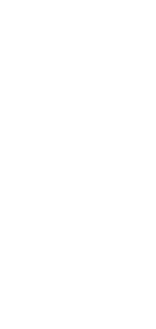

$\mathrm{Cl}^{-}(\mu \mathrm{mol} / \mathrm{L})$

$$
\begin{gathered}
\text { Control } \\
1 \mathrm{mg} / \mathrm{Kg} \text { bwt } \\
5 \mathrm{mg} / \mathrm{Kg} \text { bwt } \\
20 \mathrm{mg} / \mathrm{Kg} \text { bwt } \\
5000 \mathrm{mg} / \mathrm{Kg} \text { bwt } \\
\text { Ref. range }
\end{gathered}
$$$$
\text { ANOVA p-value }
$$

$$
\begin{gathered}
\text { Control } \\
1 \mathrm{mg} / \mathrm{Kg} \text { bwt } \\
5 \mathrm{mg} / \mathrm{Kg} \text { bwt } \\
20 \mathrm{mg} / \mathrm{Kg} \text { bwt } \\
5000 \mathrm{mg} / \mathrm{Kg} \text { bwt } \\
\text { Ref. range }
\end{gathered}
$$

\section{ANOVA p-value}

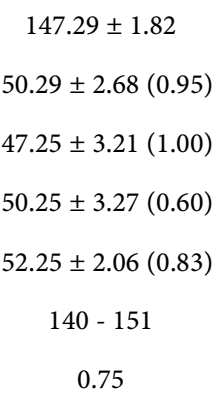

$$
7.58 \pm 0.66
$$

$16.18 \pm 2.34(0.30)$

$19.11 \pm 2.88(0.08)$

$23.02 \pm 4.03\left(0.01^{*}\right)$

$10.80 \pm 1.55(0.96)$

$3.31-4.90$

$0.01^{*}$

$$
\begin{gathered}
102.97 \pm 1.61 \\
104.44 \pm 1.21(0.89) \\
102.66 \pm 0.83(1.00) \\
104.25 \pm 0.90(0.93) \\
110.25 \pm 1.14\left(0.006^{*}\right)
\end{gathered}
$$$$
100-1007
$$

$$
0.005^{\star *}
$$

$147.25 \pm 2.50$

$147.33 \pm 3.28$

$154.50 \pm 2.90(0.70)$

$149.20 \pm 4.75(1.00)$

$149.20 \pm 4.61(1.00)$

$152.50 \pm 0.50(0.94)$

$142-151$

0.73

$$
\begin{gathered}
7.63 \pm 0.93 \\
15.06 \pm 2.05(0.74) \\
19.76 \pm 4.09(0.33) \\
22.75 \pm 5.33(0.13) \\
10.43 \pm 1.99(1.00) \\
3.82-5.55 \\
0.13
\end{gathered}
$$

$144.67 \pm 2.40(0.99)$

$145.25 \pm 4.77(1.00)$

$152.00 \pm 5.15(0.94)$

$152.00 \pm 5.00(0.96)$

$140-150$

0.66

\section{$7.50 \pm 1.32$}

$18.44 \pm 7.00(0.66)$

$18.24 \pm 4.87(0.60)$

$23.70 \pm 7.51(0.35)$

$11.26 \pm 3.18(0.99)$

$3.31-4.90$

0.36

$$
\begin{gathered}
102.78 \pm 1.26 \\
106.05 \pm 1.37(0.30) \\
104.30 \pm 0.95(0.87) \\
104.24 \pm 1.00(0.87) \\
109.8 \pm 0.3\left(0.02^{*}\right) \\
100-106 \\
0.03^{*}
\end{gathered}
$$$$
103.23 \pm 3.86
$$$$
102.30 \pm 1.57(1.00)
$$$$
101.03 \pm 0.73(0.94)
$$$$
104.27 \pm 2.04(1.00)
$$$$
110.70 \pm 2.70(0.29)
$$$$
100-107
$$

0.14

Key: () are $\mathrm{p}$ values as compared to the control group ${ }^{\star} \mathrm{p}<0.05$. Values of rats' clinical chemistry parameters are expressed as mean \pm SEM, for each dose level group and control group: $\mathrm{n}=10$ for both male and female Swiss albino rats, $\mathrm{n}=5$ for male Swiss albino rats and $\mathrm{n}=5$ for female Swiss albino rats.

\subsection{Changes in Relative Organ Weight (g/100g bwt)}

Both male and female Wistar albino rats in treated groups showed significant differences in relative weight from control groups for the kidney $(1.0 \mathrm{mg} / \mathrm{Kg}$ bwt, $\mathrm{p}=0.04 ; 20 \mathrm{mg} / \mathrm{Kg}$ bwt $\mathrm{p}<0.001$ and $5000.0 \mathrm{mg} / \mathrm{Kg}$ bwt, $\mathrm{p}=0.03)$ and heart $(20.0 \mathrm{mg} / \mathrm{Kg}$ bwt, $\mathrm{p}=0.01)$. However, there was non-significant change in the relative weights of the lungs at any dose levels in combined group of both male and female Wistar albino rats treated with OMGKRP crude aqueous suspension as compared to control. The treated male Wistar albino rats showed significant differences in relative weight from control group in kidney $(1 \mathrm{mg} / \mathrm{Kg}$ bwt, $\mathrm{p}=0.03)$ and lung $(1 \mathrm{mg} / \mathrm{Kg}$ bwt, $\mathrm{p}=0.01)$. There was no significant change between control and treated male rats in the relative organ weight of heart. The treated female Wistar albino rats showed significant differences from 
control in kidney $(20 \mathrm{mg} / \mathrm{Kg}$ bwt, $\mathrm{p}=0.03)$. However, there was no significant change in relative weight of heart and lungs at all dose levels in treated female Wistar albino rats as compared to control groups. Also, there was no significant change in relative weight of liver at all dose levels in both treated males and females rats as compared to control. The maximum effect (changes in relative organ weight) of OMGKRP crude aqueous suspension was observed on kidney and heart in both sexes and in female rats treated group at intermediate dose level of $20.0 \mathrm{mg} / \mathrm{Kg}$ bwt (Table 2).

Table 2. Changes in relative organ weight (g/100g bwt) of Wistar albino rats following daily dosing OMGKRP crude aqueous suspension for 28 days (sub-acute toxicity study).

\begin{tabular}{|c|c|c|c|c|}
\hline \multicolumn{2}{|c|}{ Organ } & \multicolumn{3}{|c|}{ Relative organ weight changes ( $\mathrm{g} / 100 \mathrm{~g}$ body weight) } \\
\hline & Group & Both male \& female & Male & Female \\
\hline \multirow{6}{*}{ Heart } & Control & $0.36 \pm 0.02$ & $0.37 \pm 0.03$ & $0.34 \pm 0.02$ \\
\hline & $1 \mathrm{mg} / \mathrm{Kg}$ bwt & $0.41 \pm 0.01(0.57)$ & $0.40 \pm 0.02(0.97)$ & $0.43 \pm 0.02(0.55)$ \\
\hline & $5 \mathrm{mg} / \mathrm{Kg}$ bwt & $0.43 \pm 0.02(0.33)$ & $0.44 \pm 0.02(0.73)$ & $0.42 \pm 0.03(0.61)$ \\
\hline & $20 \mathrm{mg} / \mathrm{Kg}$ bwt & $0.50 \pm 0.02\left(0.01^{\star}\right)$ & $0.48 \pm 0.01(0.24)$ & $0.49 \pm 0.05(0.06)$ \\
\hline & $5000 \mathrm{mg} / \mathrm{Kg}$ bwt & $0.48 \pm 0.09(0.10)$ & $0.52 \pm 0.20(0.25)$ & $0.43 \pm 0.03(0.65)$ \\
\hline & ANOVA values & $0.01^{*}$ & 0.16 & 0.11 \\
\hline \multirow{6}{*}{ Kidney } & Control & $0.38 \pm 0.02$ & $0.37 \pm 0.02$ & $0.40 \pm 0.05$ \\
\hline & $1 \mathrm{mg} / \mathrm{Kg}$ bwt & $0.46 \pm 0.02\left(0.04^{*}\right)$ & $0.48 \pm 0.02\left(0.03^{*}\right)$ & $0.44 \pm 0.01(0.84)$ \\
\hline & $5 \mathrm{mg} / \mathrm{Kg}$ bwt & $0.45 \pm 0.01(0.14)$ & $0.44 \pm 0.01(0.23)$ & $0.45 \pm 0.01(0.69)$ \\
\hline & $20 \mathrm{mg} / \mathrm{Kg}$ bwt & $0.49 \pm 0.02(<0.001)$ & $0.45 \pm 0.02(0.11)$ & $0.54 \pm 0.03\left(0.03^{*}\right)$ \\
\hline & $5000 \mathrm{mg} / \mathrm{Kg}$ bwt & $0.49 \pm 0.05\left(0.03^{*}\right)$ & $0.48 \pm 0.09(0.11)$ & $0.50 \pm 0.07(0.34)$ \\
\hline & ANOVA values & $0.004^{*}$ & $0.04^{*}$ & $0.04^{*}$ \\
\hline \multirow{6}{*}{ Lung } & Control & $0.74 \pm 0.07$ & $0.63 \pm 0.04$ & $0.88 \pm 0.12$ \\
\hline & $1 \mathrm{mg} / \mathrm{Kg}$ bwt & $0.89 \pm 0.05(0.66)$ & $0.89 \pm 0.07\left(0.01^{*}\right)$ & $0.89 \pm 0.07(1.00)$ \\
\hline & $5 \mathrm{mg} / \mathrm{Kg}$ bwt & $0.93 \pm 0.09(0.43)$ & $0.74 \pm 0.04(0.49)$ & $1.11 \pm 0.15(0.73)$ \\
\hline & $20 \mathrm{mg} / \mathrm{Kg}$ bwt & $0.86 \pm 0.07(0.80)$ & $0.74 \pm 0.03(0.48)$ & $1.00 \pm 0.13(0.96)$ \\
\hline & $5000 \mathrm{mg} / \mathrm{Kg}$ bwt & $0.92 \pm 0.09(0.68)$ & $0.79 \pm 0.07(0.42)$ & $1.06 \pm 0.11(0.94)$ \\
\hline & ANOVA values & 0.50 & $0.02^{*}$ & 0.67 \\
\hline \multirow{6}{*}{ Liver } & Control & $4.08 \pm 0.14$ & $4.06 \pm 0.18$ & $4.10 \pm 0.28$ \\
\hline & $1 \mathrm{mg} / \mathrm{Kg}$ bwt & $4.05 \pm 0.11(1.00)$ & $3.95 \pm 0.09(0.99)$ & $4.21 \pm 0.27(1.00)$ \\
\hline & $5 \mathrm{mg} / \mathrm{Kg}$ bwt & $3.87 \pm 0.11(0.86)$ & $3.67 \pm 0.11(0.56)$ & $4.06 \pm 0.16(1.00)$ \\
\hline & $20 \mathrm{mg} / \mathrm{Kg}$ bwt & $4.29 \pm 0.16(0.84)$ & $4.03 \pm 0.23(1.00)$ & $4.60 \pm 0.11(0.52)$ \\
\hline & $5000 \mathrm{mg} / \mathrm{Kg}$ bwt & $4.09 \pm 0.31(1.00)$ & $3.74 \pm 0.01(0.86)$ & $4.45 \pm 0.58(0.90)$ \\
\hline & ANOVA values & 0.31 & 0.46 & 0.33 \\
\hline
\end{tabular}

Key: ()$=p$ values as compared to the control group. Relative organ weights are expressed as mean \pm SEM, $n=10$ per group for both male and female, $n=5$ per group for male and $\mathrm{n}=5$ per group for female, ${ }^{*} \mathrm{p}<0.05$. 


\subsection{Histopathological Analysis of the Effect of OMGKRP on the Kidney, Lung and Heart Tissues of Wistar Albino Rats}

The histopathological analyses of lungs (Figure 1) showed that at all dose levels there was severe diffuse interstitial type 2 pneumocytes cells proliferation with large lymphocytes and other inflammatory, and phagocytic cells (eosinophil, macrophages) infiltration. However, cellular infiltrations were observed in the peri-bronchiolar area as well as accumulation of cellular debris in some bronchioles, moderate nodular hyperplasia of bronchiolar epithelial cells and moderate to severe vascular congestion were observed. Furthermore, mild perivascular edema and accumulation of cellular exudate were observed in some bronchioles. For histopathological analyses of kidneys, findings showed no morphological

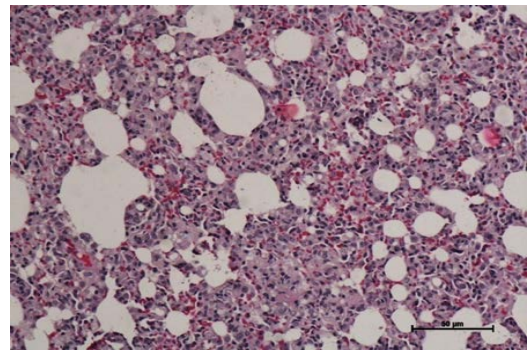

(a)

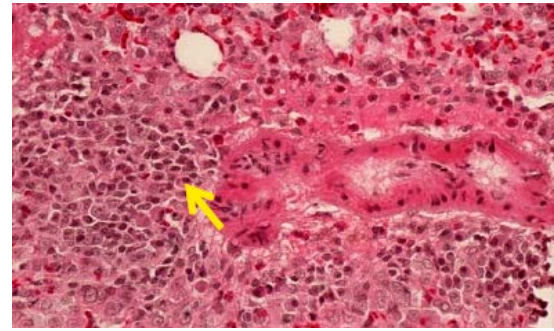

(c)

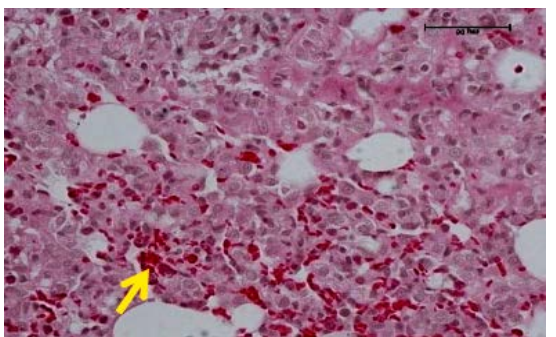

(e)

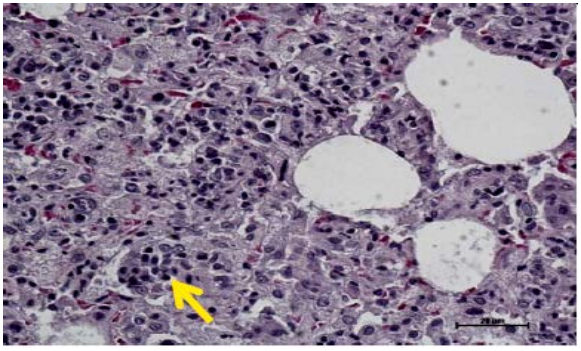

(b)

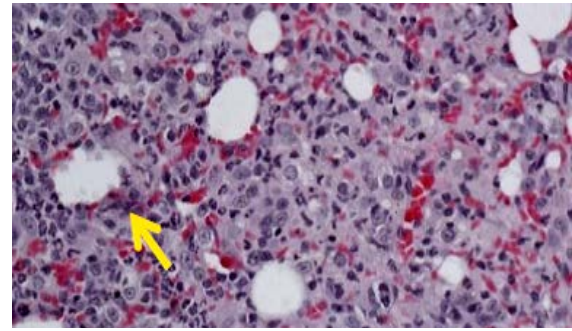

(d)

Figure 1. Photomicrographs of Wistar albino rat lung tissues following the administration of the different sub-acute doses of OMGKRP. (a) Normal lung tissue (control); (b) sub-acute doses of $1.0 \mathrm{mg} / \mathrm{Kg}$ bwt; (c) sub-acute dose of 5.0 $\mathrm{mg} / \mathrm{Kg}$ bwt; (d) sub-acute dose of $20.0 \mathrm{mg} / \mathrm{Kg}$ bwt; (e) sub-acute dose of 5000.0 $\mathrm{mg} / \mathrm{Kg}$ bwt. Arrows key: (a) normal lung tissue; (b) lung with diffuse severe interstitial cells (type II pneumocytes) proliferation; (c) severe diffuse interstitial pneumonia and peri-bronchiolar eosinophils and mononuclear cells $\times 40$ and (d) lung with type 2 pneumocytes hyperplasia; PMN cells infiltrations $\times 40$ and (e) severe proliferation type 2 pneumocytes $\times 40$. 
changes in treated Wistar albino rats. In all groups, renal cortex and renal corpuscles were preserved, as well as all types of renal tubules. However, there were dose-depended moderate to severe diffuse vascular congestion and multifocal interstitial mononuclear inflammatory cells infiltrations especially for the limit dose of $5000.0 \mathrm{mg} / \mathrm{Kg}$ bwt (Figure 2). Brown pigmentation depositions were observed in cytoplasm of renal tubular cells at dose level of $20.0 \mathrm{mg} / \mathrm{Kg}$ bwt. The histopathological analysis of liver (Figure 3), revealed mild congestion, diffuses centri-lobular hepatic cord atrophy and sinusoid dilation. Mild multifocal peri-portal mononuclear inflammatory cells infiltrations (reactive hepatitis) and multifocal random typhoid like granulomas (macrophage accumulation) were observed at lower dose of $1.0 \mathrm{mg} / \mathrm{Kg}$ bwt, intermediate at $20.0 \mathrm{mg} / \mathrm{Kg}$ bwt and at high dose of $5000.0 \mathrm{mg} / \mathrm{Kg}$ bwt. The histopathological analysis of heart revealed mild congestion and mild myocardial fiber atrophy at dose level of $1.0 \mathrm{mg} / \mathrm{Kg}$ bwt and mild myocardial fiber hypertrophy at dose level of $20.0 \mathrm{mg} / \mathrm{Kg}$ bwt (Figure 4).

\section{Discussion}

Poisoning of human and animals is a global public health challenge especially with OMGKR in Goma city located in Eastern Democratic Republic of Congo (DRC) causing damages to various body organs, serum enzymes and blood chemistry of the victim. The study findings showed that there was no acute toxicity

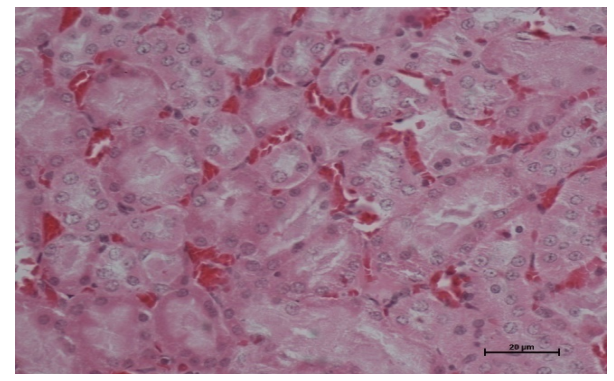

(a)

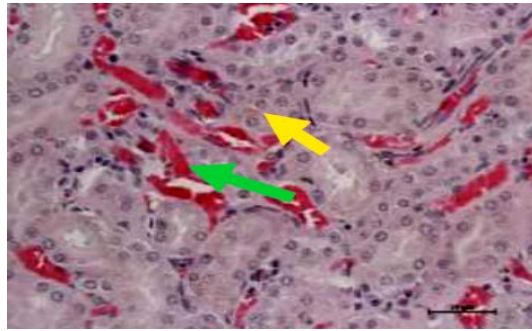

(b)

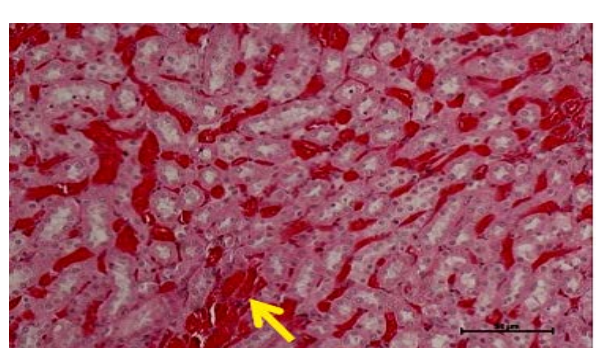

(c)

Figure 2. Photomicrographs of Swiss albino rat kidney tissues following the administration of the different sub-acute doses of OMGKRP. (a) Normal kidney tissue (control); (b) sub-acute dose of $20.0 \mathrm{mg} / \mathrm{Kg}$ bwt; (c) sub-acute dose of $5000.0 \mathrm{mg} / \mathrm{Kg}$ bwt. Arrows key: (a) normal kidney tissue (control); (b) moderate congestion (green) and brown pigmentation deposition in cytoplasm (yellow) of renal tubular cells; (c) diffuse severe congestion $\times 20$. 


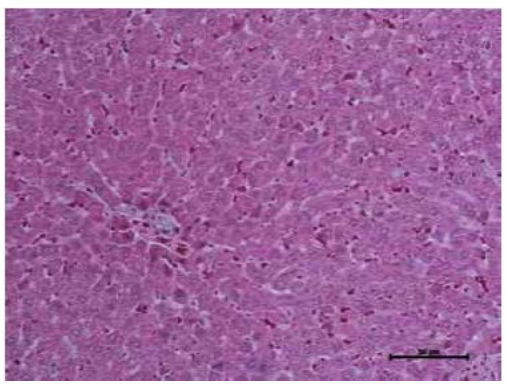

(a)

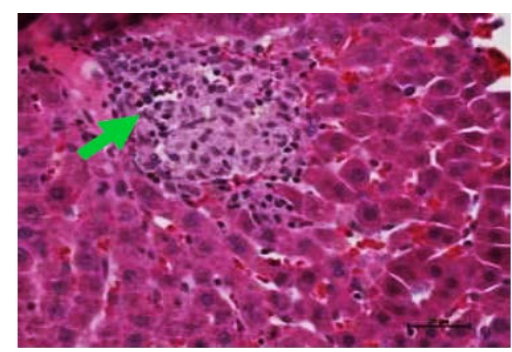

(c)

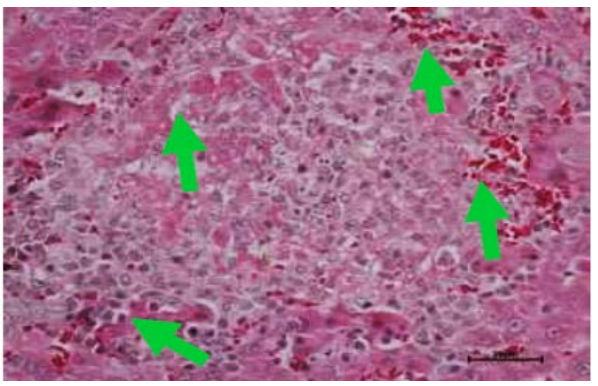

(b)

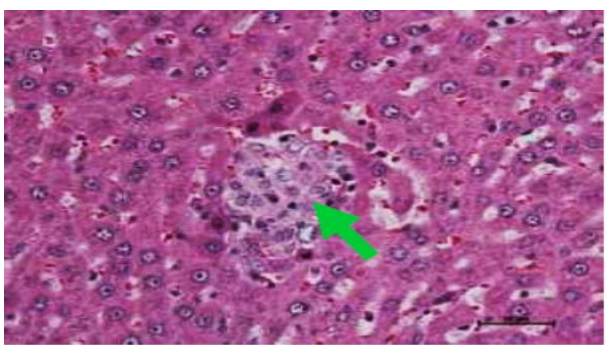

(d)

Figure 3. Photomicrographs of Swissalbino rat liver tissue following the administration of the different sub-acute doses of OMGKRP. (a) Normal Liver tissue (Control); (b) sub-acute dose of $1.0 \mathrm{mg} / \mathrm{Kg}$ bwt; (c) sub-acute dose of $5.0 \mathrm{mg} / \mathrm{Kg}$ bwt; (d) sub-acute dose of $20.0 \mathrm{mg} / \mathrm{Kg}$ bwt. Arrows key: (a) normal liver tissue (Control); (b) random multifocal necrosis $\times 40$; (c) typhoid like granuloma with lymphocyte infiltration $\times 40$ and (d) clear typhoid-like granuloma $\times 40$.

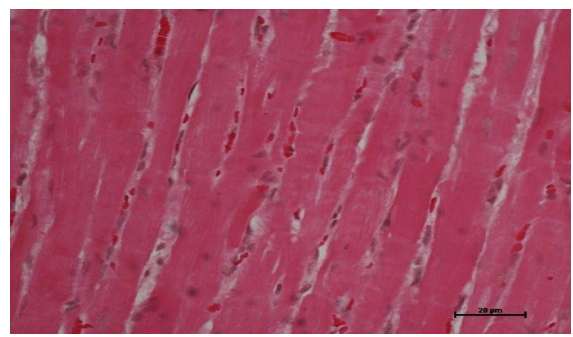

(a)

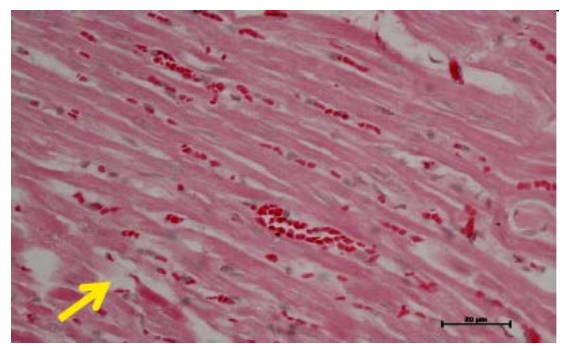

(b)

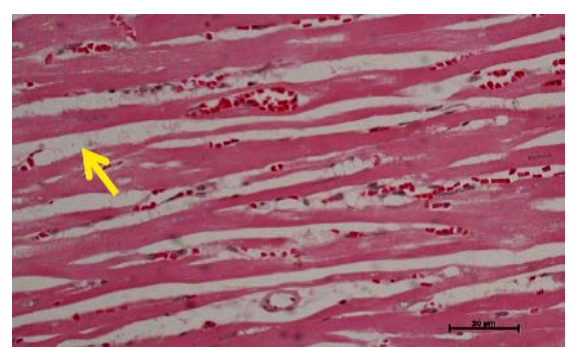

(c)

Figure 4. Photomicrographs of Wistar albino rat heart tissues following the administration of the different sub-acute doses of OMGKRP. (a) Normal heart tissue (Control); (b) sub-acute dose of $1.0 \mathrm{mg} / \mathrm{Kg}$ bwt; (c) sub-acute dose of 20.0 $\mathrm{mg} / \mathrm{Kg}$ bwt. Arrows Key: (a) normal heart muscle tissue (control); (b) mild myocardium fibers atrophy $\times 40$ and (c) mild myocardial fibers hypertrophy $\times 40$. 
observed within 24 hours of administration of the different dose levels of OMGKRP Karuho poison (no death). And these findings are similar to what is observed and reported among the victims where no death is immediately reported when such people are exposed to these poisons. However, prolonged exposure (sub-acute toxicity), the findings showed body weight loss, shortness of breath, reduced food and water intake and piloerection among the experimental animals at the different dose levels. These signs and symptoms are similar to what has been reported or observed among the victims in the communities who are exposed to these Karuho poisons chronically and thus showing signs and symptoms that are occasionally confused with those of tuberculosis, typhoid, HIV/AIDS and many other conditions.

The finding from the study showed that OMGKRP poison caused damage to the renal, pulmonary, liver and cardiac tissues especially at the dose level of 20.0 $\mathrm{mg} / \mathrm{Kg}$ bwt. However, there were no severe effects observed on the organs with the high limit dose level of $5000.0 \mathrm{mg} / \mathrm{Kg}$ bwt as compared to the low dose level of 1.0, 5.0 and $20.0 \mathrm{mg} / \mathrm{Kg}$ bwt. These findings were similar to what is observed with some other chemical substances that are more poisonous in dilute form or low dose as compard to the concentrated form and these findings could be due to their ability to be highly absorbed in more dilute form than the concentrated form in the gastrointestinal tract (GIT). Indeed, Wistar albino rats treated by sub-acute doses of OMGKRP were associated with abnormalities in serum biochemical parameters including total protein, albumin, creatinine, urea, potassium, and direct bilirubin and chloride levels. The total proteins measurements are commonly used in the diagnosis and treatment of a variety of diseases involving the liver, kidney, lung as well as other metabolic disorders. It has been reported that in inflammatory conditions such as that produced by Karuho poison, the liver responds by producing a large number of acute-phase proteins including C-reactive protein (CRP), mannose-binding protein, complements factors, ferritin, ceruloplasmin, serum amyloid $\mathrm{A}$ and haptoglobin and by decreasing negative phase proteins such as albumin [31]. Also, it has been reported that the binding of xenobiotics to albumin reduce serum albumin level [31]. Treated rats of both sexes with OMGKRP were associated with significantly increased total protein level at lower dose of $1 \mathrm{mg} / \mathrm{Kg}$ bwt and intermediate dose level of 5.0 and $20.0 \mathrm{mg} / \mathrm{Kg}$ bwt; and with significantly decreased serum albumin level at high dose of $5000 \mathrm{mg} / \mathrm{Kg}$ bwt at day 28. Indeed, hyperproteinemia is an indication of dehydration, inflammation, hepatotoxicity and primary glomerular disease [31] which may be associated with this poison. Also, it has been reported that in interstitial lung disease caused by toxicants, there is increase in surfactant proteins [32]. This suggests that the OMGKRP had an inflammatory effect on the lung, kidney or liver, hence the hyperproteinemia observed in the study.

The urea and creatinine levels are compounds derived from proteins metabolism which are eliminated by the kidney. In the present work, increment of urea levels more than normal range (Table 1), is a relevant indicator for renal im- 
pairment [33] and was observed in the findings. The elevated creatinine levels is a reliable indicator of impaired glomerular filtration or alterations in renal blood flow and severe tubular dysfunction along with urea [33]. In fact, the urea levels increased at all dose levels in both male and female rats and was increasingly significant at intermediate dose level of $20 \mathrm{mg} / \mathrm{Kg}$ bwt. There was significant difference in increase between control and both male and treated female rat's serum creatinine and urea levels. The increase in creatinine levels were recorded at lower dose of $1 \mathrm{mg} / \mathrm{Kg}$ bwt and intermediate dose level of $20 \mathrm{mg} / \mathrm{Kg}$ bwt in both male and female rats. However, normal level of serum creatinine and urea was recorded at higher dose level of $5000 \mathrm{mg} / \mathrm{Kg}$ bwt. Increase in the urea and creatinine levels conjointly with increased total proteins concentration directly suggests impaired glomerular filtration or alterations in renal blood flow and severe tubular dysfunction induced by OMGKRP aqueous suspension. This was also observed in the histopathology study of the poisoned rats.

The increased potassium levels can lead to significant hemodynamic and neurologic consequences, whereas levels exceeding $8.5 \mathrm{mEq} / \mathrm{L}$ can cause respiratory paralysis or cardiac arrest and can quickly be fatal. Occasionally, patients may report the symptoms including frank muscle paralysis, dyspnea, palpitations, chest pain, nausea or vomiting and paresthesia [34]. Hyperkalemia can result from increased potassium intake, decreased potassium excretion, or a shift of potassium from the intracellular to the extracellular space [34]. The most common causes of increased blood potassium levels, involve the decreased renal potassium excretion in conditions of very low levels, of aldosterone, low sodium levels delivery to the distal tubule, low urine flow, low serum potassium level and kidney injury [34]. The findings revealed serum potassium levels significantly increased in both male and female treated rats at dose level of $20 \mathrm{mg} / \mathrm{Kg}$ bwt. Also, observed was that serum potassium level in control group was slightly higher than that of the reference range values that could suspect an artifact issue. However, observed was that serum potassium levels were increased with dose levels in the treated group of Swiss albino rats. This suggested that increase in blood potassium indicates that OMGKRP affected kidney urine flow. The elevations in chloride levels may be associated with certain kidney diseases like the Type 2 renal tubular acidosis, Type 1 tubular acidosis and over activity of the parathyroid glands. Hyperchloremia can be symptomatic with signs of Kussmaul's breathing, weakness, and intense thirst [35]. Both treated male and female rats were associated with significantly increased serum chloride levels but only at a high dose level of $5000 \mathrm{mg} / \mathrm{Kg}$ bwt. Hyperchloremia suggests that Karuho crude aqueous suspension is since it affects the kidneys. And the damage to the kidney could most probably be due to heavy metals such as Mercury, lead, arsenic compounds and many others that may also be used in the Karuho poison preparation.

The organ weight is an index of swelling, atrophy or hypertrophy [36]. The relative organ weight is fundamental in the diagnosis of organ injury. Heart, kidneys, and lungs are also primary organs affected by metabolic reactions 
caused by toxicants like liver and spleen. The study findings for both male and female treated rats were associated with significantly increased relative kidney weight at all dose levels and significantly increased heart weight at intermediate dose level of $20.0 \mathrm{mg} / \mathrm{Kg}$ bwt. The increase in relative lung weight was significantly lower at a dose level of $1.0 \mathrm{mg} / \mathrm{Kg}$ bwt. Actually, blood chemistry assessment showed that the OMGKRP had an effect on the kidney, liver and lung in the study animals. Histopathological examinations of the selected organs including kidney, liver, lung and heart were performed to provide information to strengthen the findings on biochemical parameters and relative organ weight. Indeed, histopathological examination of the kidneys of treated rats with OMGKRP crude aqueous suspension revealed dose-depended severe diffuse congestion of kidney and brown pigmentation in cytoplasm renal tubular cell at intermediate dose level of $20.0 \mathrm{mg} / \mathrm{Kg}$ bwt. The histopathological findings of kidney and elevated serum creatinine, chloride, total proteins, chloride and decreased albumin levels show that OMGKRP crude aqueous suspension had a toxic effect on kidney functions especially impairment of glomerular filtration, and alteration in renal blood flow and tubular dysfunction. The histopathological analysis of liver tissues revealed no significant change in relative weight of liver at all dose levels in both treated males and females rats as compared to control. However, photomicrographs (Figure 3(c) and Figure 3(d)), showed random multifocal necrosis and clear typhoid-like granuloma with lymphocyte infiltration. Furthermore, treated male rats showed significant difference from control group in direct bilirubin at dose level of $20 \mathrm{mg} / \mathrm{Kg}$ bwt. Also, there was dose-depended effect in treated group in biochemical liver function tests including GGT, ALT and AST levels. However, no significant difference was observed in these parameters as compared to the control group. Anyway, pathological and biochemical findings showed that OMG KRP has deleterious effects on liver tissues.

The histopathological analysis of lung of treated rats revealed that at all dose levels, there was severe diffuse interstitial type 2 pneumocytes cells proliferation with large lymphocytes and other inflammatory and phagocytic cells (eosinophil, macrophages). Also, infiltrations in the peri-bronchiolar area, accumulation of cellular debri in some bronchioles, moderate nodular hyperplasia of bronchilor epithelial cells, mild perivascular edema of bronchioles and moderate to severe vascular congestion were observed. This led to observed interstitial or atypical pneumonia in treated Wistar albino rats by OMGKRP crude aqueous suspension. The above findings were correlated to signs and symptoms including chest discomfort, cough and shortness of breath claimed to be associated with Karuho poisoning where the signs and symptoms are often confused with those of TB in Goma city as reported by Simisi [37]. It has been reported that disorders of respiratory system from chronic alveolar hypoxemia such as those caused by OMGKRP poison, hence leading to pulmonary hypertension. Then pulmonary arterial hypertension can result in right ventricular enlargement (hypertrophy or dilatation) and thus over time leading to right heart failure. This 
condition is called cor-pulmonale [38]. Furthermore, recent findings revealed that congestive heart failure is found in about one-quarter of cases of chronic kidney disease and that most common cause of congestive heart failure is ischemic heart disease [39]. The prevalence of congestive heart failure increases greatly as the patient's renal function deteriorates, and, at end-stage renal disease, can reach about $65 \%-70 \%$. There is mounting evidence that chronic kidney disease itself is a major contributor to severe cardiac damage and, conversely, that congestive heart failure is a major cause of progressive chronic kidney diseases [39]. The claim is that victims of Karuho poisoning are associated with renal, pulmonary, liver and cardiac manifestation [2]. Actually, histopathological findings have confirmed the damages to these organs by OMGKRP Karuho poison. Indeed, histopathological analysis of treated rats showed moderate to severe vascular congestion of heart and mild myocardial fiber atrophy at dose level of 1 $\mathrm{mg} / \mathrm{Kg}$ bwt. The mild to severe congestion was time and dose-depended. Severe congestion was observed in satellites groups kept 14 days after sub-acute doses. The findings suggest that OMGKRP crude aqueous suspension may be associated with lung obstructive disease that induce congestive heart failure and cardiac damage (ischemia) resulting in renal dysfunction. Nevertheless, heart failure and cardiac damage could likely be caused by chronic kidney dysfunction induced by direct effect of the poison on the kidney. And since the major organ affected was the kidney, it was possible that heavy metals such as mercury, lead, copper, arsenic, glass powder and many others were also used in the OMGKRP poison preparation or these metals were present in high amounts in the raw materials that were used in the preparation of the poison.

\section{Conclusion}

Sub-acute doses of OMGKRP Karuho poison in Wistar albino rats, a poison used by unscrupulous individuals to cause homicide in Goma city, were associated with kidney, pulmonary, liver and heart tissue damages as well as causing changes in blood chemistry and serum enzymes. Furthermore, OMGKRP induced severe diffuse interstitial type 2 pneumocytes cells proliferation with large lymphocytes and other inflammatory and phagocytic cells (eosinophil, macrophages) with infiltrations in the peri-bronchiolar area, accumulation of cellular debri in some bronchioles, moderate nodular hyperplasia of bronchiolar epithelial cells, mild perivascular edema of bronchioles and moderate to severe vascular congestion. In the heart, it caused mild myocardial fibers hypertrophy while in the liver tissues; the damage was associated with random multifocal necrosis and clear typhoid-like granuloma with lymphocyte infiltration.

\section{Acknowledgements}

I am grateful to all staff members of the Department of Pharmacology \& Therapeutics Makerere University College of Health Science. All Ethical committee of Makerere University, Universitelibre des pays des grandslacs (ULPGL) Goma, 
DRC and to the Directorate of Health Services in Goma city/DRC. I am also very grateful to my study participants and my research assistants.

\section{Conflicts of Interest}

The authors declare no conflicts of interest.

\section{References}

[1] Jesslin, J., Adepu, R. and Churi, S. (2010) Assessment of Prevalence and Mortality Incidences Due to Poisoning in a South Indian Tertiary Care Teaching Hospital. Indian Journal of Pharmaceutical Sciences, 72, 587-591.

https://doi.org/10.4103/0250-474X.78525

[2] Uwizeye, O. (2004) Ethnobotanical Survey of Plants Used against Poisons "Karuho" in Goma Health District. Universite libre des pays des grands lacs (ULPGL), 80.

[3] Kaboru, B.B. and Namegabe, E.N. (2014) Geographical, Health Systems and Sociocultural Patterns of Tb/Hiv Co-Infected Patients Health Seeking Behavior in a Conflict Affected Setting: The Case of Eastern Democratic Republic of Congo. Journal of Community Medicine \& Health Education, 4, 263.

https://doi.org/10.4172/2161-0711.1000263

[4] IRIN (2009) Toxic Toad Scam Killing Patients: Health News, GOMA. http://www.irinnews.org/report/84354/drc-toxic-toad-scam-killing-patients

[5] Trancă, S.D., Szabo, R. and Mihaela Cociş, M. (2017) Acute Poisoning Due to Ingestion of Datura stramonium-A Case Report. Romanian Journal of Anesthesia and Intensive Care, 24, 65-68. https://doi.org/10.21454/rjaic.7518.241.szb

[6] Quintero, E., Thessen, A.E., Arias-Caballero, P. and Ayala-Orozco, B. (2014) A Statistical Assessment of Population Trends for Data Deficient Mexican Amphibians. Peer Journal, 2, e703. https://doi.org/10.7717/peerj.703

[7] Yotsu-Yamashita, M., Kim, Y.H., Dudley, S.C., Choudhary, G., Pfahnl, A., Oshima, Y. and Daly, J.W. (2004) The Structure of Zetekitoxin AB, a Saxitoxin Analog from the Panamanian Golden Frog Atelopus zeteki: A Potent Sodium-Channel Blocker. Proceedings of the National Academy of Sciences, USA, 101, 4346-4351. https://doi.org/10.1073/pnas.0400368101

[8] Pettit, G.R., Kamano, Y., Drasar, P., Inoue, M. and Knight, J.C. (1987) Steroids and Related Natural Products. 104. Bufadienolides. 36. Synthesis of Bufalitoxin and Bufotoxin. Journal of Organic Chemistry, 15, 3573-3578.

https://doi.org/10.1021/jo00392a014

[9] Pantanowitz, L., Naude, T.W. and Leisewitz, A. (1998) Noxious Toads and Frogs of South Africa. South African Medical Journal, 88, 1408-1414. https://www.ajol.info/index.php/samj/article/download/149345/138844

[10] Balazs, T., Hanig, J.P. and Herman, E.H. (1986) Toxic Responses of the Cardiovascular System. In: Klaassen, C.D., Amdur, M.O. and Doull, J., Eds., Casarett and Doulp s Toxicology: The Basic Science of Poisons, Third Edition, Macmillan Publishing Company, New York, 387-411.

[11] Uddman, R., Goadsby, P.J., Jansen-Olesen, I. and Edvinsson, L. (1999) Helospectin-Like Peptides: Immunochemical Localization and Effects on Isolated Cerebral Arteries and on Local Cerebral Blood Flow in the Cat. Journal of Cerebral Blood Flow and Metabolism, 19, 61-67.

https://doi.org/10.1097/00004647-199901000-00007

https://journals.sagepub.com/doi/pdf/10.1097/00004647-199901000-00007 
[12] Nobile, M., Noceti, F., Prestipino, G. and Possani, L.D. (1996) Helothermine, a Lizard Venom Toxin, Inhibits Calcium Current in Cerebellar Granules. Experimental Brain Research, 110, 15-20. https://doi.org/10.1007/BF00241369

[13] Hendon, R.A. and Tu, A.T. (1981) Biochemical Characterization of the Lizard Toxin Gilatoxin. Biochemistry, 20, 3517-3522. https://doi.org/10.1021/bi00515a033

[14] White, J. and Weinstein, S.A. (2015) Reply to Vikrant and Verma about "Monitor Lizard Envenoming". Renal Failure, 37, 740-741. https://doi.org/10.3109/0886022X.2015.1006116

[15] Cantrell, F.L. (2003) Envenomation by the Mexican Beaded Lizard: A Case Report. Journal of Toxicology: Clinical Toxicology, 41, 241-244. https://doi.org/10.1081/CLT-120021105

[16] Bou-Abboud, C.F. and Kardassakis, D.G. (1988) Acute Myocardial Infarction Following a Gila Monster (Heloderma Suspectum Cinctum) Bite. Western Journal of Medicine, 148, 577.

[17] Mebs, D. (1973) Chemistry of Animal Venoms, Poisons and Toxins. Cellular and Molecular Life Sciences, 29, 1328-1334. https://doi.org/10.1007/BF01922799

[18] EFSA (2008) Tropane Alkaloids (from Datura sp.) as Undesirable Substances in Animal Feed: Scientific Opinion of the Panel on Contaminants in the Food Chain. The European Food Safety Authority (EFSA) Journal, 691, 1-55. https://pdfs.semanticscholar.org/a969/8e3040ec262813b6a79884872bde4639fc11.pd f

[19] Kohnen-Johannsen, K.L. and Oliver Kayser, O. (2019) Tropane Alkaloids: Chemistry, Pharmacology, Biosynthesis and Production. Molecules, 24, 796. https://doi.org/10.3390/molecules24040796

[20] Bushnell, D.A., Cramer, P. and Kornberg, R.D. (2002) Structural Basis of Transcription: $\alpha$-Amanitin-RNA Polymerase II Cocrystal at $2.8 \AA$ Resolution. Proceedings of the National Academy of Sciences, USA, 99, 1218-1222. https://doi.org/10.1073/pnas.251664698

[21] Cooper, J.A. (1987) Effects of Cytochalasin and Phalloidin on Actin. The Journal of Cell Biology, 105, 1473-1478. https://doi.org/10.1083/jcb.105.4.1473

[22] Tchounwou, P.B., Yedjou, C.G., Patlolla, A.K. and Sutton, D.J. (2012) Heavy Metal Toxicity and the Environment. Experientia Supplementum, 101, 133-164. https://doi.org/10.1007/978-3-7643-8340-4_6

[23] Kyolo, S.K., Bbosa, G.S., Odda, J., Lubega, A.M. and Namegabe, N.E. (2018) Toxicity Profile of Karuho Poison on the Brain of Wistar Albino Rats. Neuroscience \& Medicine, 9, 63-80. https://www.scirp.org/pdf/NM_2018061414572404.pdf https://doi.org/10.4236/nm.2018.92008

[24] NRC (2011) Prudent Practices in the Laboratory-Handling and Management of Chemical Hazards. National Research Council (NRC) Committee on Prudent Practices in the Laboratory. National Academies Press (USA), Washington DC. https://www.ncbi.nlm.nih.gov/books/NBK55878/pdf/Bookshelf_NBK55878.pdf

[25] Ghosh, M.M. (2007) Fundamentals of Experimental Pharmacology. Indian Journal of Pharmacology, Kolkata-India, 39, 216.

[26] OECD (2001) Acute Oral Toxicity Fixed Dose Procedure: OECD/OCDE 420. Organization for Economic Co-Operation and Development (OECD), Paris, 1-14. https://ntp.niehs.nih.gov/iccvam/suppdocs/feddocs/oecd/oecd_gl420.pdf

[27] NIH (2011) Guide for the Care and Use of Laboratory Animals. 8th Edition, National Research Council of the National Academies Press, Washington DC.

http://grants.nih.gov/grants/olaw/Guide-for-the-care-and-use-of-laboratory-animal 
s.pdf

[28] OECD (2008) Repeated Dose 28 Day Oral Toxicity Study in Rodents: OECD/OCDE 407. Organization for Economic Co-Operation and Development (OECD), Paris, 1-13. https://ntp.niehs.nih.gov/iccvam/suppdocs/feddocs/oecd/oecdtg407-2008.pdf

[29] Raghuramulu, N., Madhavan, K. and Kalyanasundaram, S. (2003) A Manual of Laboratory Techniques. 2nd Edition, Indian Council of Medical Research (ICMR), New Delhi, 1-424.

https://www.icmr.nic.in/content/manual-laboratory-techniques-second-edition-200 3-nraghuramulu-kmadhavan-skalyanasundaram

[30] Bhardwaj, S. (2014) Elimination of Young Erythrocytes from Blood Circulation and Altered Erythropoietic Patterns during Paraquat Induced Anemic Phase in Mice. PLOS ONE, 9, e99364. https://doi.org/10.1371/journal.pone.0099364

[31] Evans, G.O. (2009) Animal Clinical Chemistry: A Practical Handbook for Toxicologists and Biomedical Researchers. 2nd Edition, CRC Press, Taylor \& Francis Group, Boca Raton, 1-368. https://doi.org/10.1201/9781420080124

https://www.crcpress.com/Animal-Clinical-Chemistry-A-Practical-Handbook-forToxicologists-and-Biomedical/Evans/p/book/9781420080117

[32] Inomata, S., Takahashi, H., Nagata, M., Yamada, G., Shiratori, M., Tanaka, H., et al. (2004) Acute Lung Injury as an Adverse Event of Gefitinib. Anticancer Drugs, 15, 461-467. https://doi.org/10.1097/01.cad.0000127666.12215.7b

[33] Vidal, A., Fallarero, A., Peña, B.R., Medina, M.E., Gra, B., Rivera, F., et al. (2003) Studies on the Toxicity of Punica granatum L. (Punicaceae) Whole Fruit Extracts. Journal of Ethnopharmacology, 89, 295-300. https://doi.org/10.1016/j.jep.2003.09.001

[34] Bond, E.F. (2000) Channelopathies: Potassium-Related Periodic Paralyses and Similar Disorders. AACN Advanced Critical Care, 11, 261-270. https://doi.org/10.1097/00044067-200005000-00009

[35] Cambier, C., Detry, B., Beerens, D., Florquin, S., Ansay, M., Frans, A., et al. (1998) Effects of Hyperchloremia on Blood Oxygen Binding in Healthy Calves. Journal of Applied Physiology, 85, 1267-1272. https://doi.org/10.1152/jappl.1998.85.4.1267

[36] Amresh, G., Singh, P. and Rao, C. (2008) Toxicological Screening of Traditional Medicine Laghupatha (Cissampelos pareira) in Experimental Animals. Journal of Ethnopharmacology, 116, 454-460. https://doi.org/10.1016/j.jep.2007.12.008

[37] Simisi, K. (2000) Contribution à l'étude des plantes médicinales utilisées contre l'empoisonnement dans la collectivité de Bwisha, territoire de RutsuruFaculty of Medicine, University of Goma/DRC.

http://www.congo-autrement.com/page/sante/le-saviez-vous-le-charbon-makala-un -anti-poison-ultra-puissant.html

[38] Weitzenblum, E. (2003) Chronic Cor Pulmonale. Heart, 89, 225-230. https://doi.org/10.1136/heart.89.2.225

[39] Silverberg, D., Wexler, D., Blum, M., Schwartz, D. and Iaina, A. (2004) The Association between Congestive Heart Failure and Chronic Renal Disease. Current Opinion in Nephrology and Hypertension, 13, 163-170.

https://doi.org/10.1097/00041552-200403000-00004 